\title{
Plan de formación e inserción profesional del INEM. Instituto Nacional de Empleo
}

El Estado Español, en 1978, reordena sus organismos de tal manera que problemas complejos como los del empleo, cuyas funciones hasta esa fecha estaban asignadas a la Seguridad Social y que no son propias de la misma porque suponen un acotamiento del tema a la vertiente de prestaciones, salgan de ella. El concepto de servicio público se hace presente en la creación de un Organismo específico -INEM (Instituto Nacional de Empleo-_ que no direccione el empleo, la formación profesional y la cobertura del desempleo en facetas de actuación distintas y haga operativas y congruentes las medidas de una politica integral de empleo. Hasta esa fecha la ordenación de los servicios de colocación estaba encomendada de forma fraccionada a diversos Ministerios, lo que dificultaba una política y toma de decisiones unificada y una respuesta rápida y eficaz.

Al INEM se le da el carácter de Organismo Autónomo Administrativo, con personalidad jurídica propia para el cumplimiento de sus,fines y adscrito al Ministerio de Trabajo y Seguridad Social, con amplias funciones de asistencia a trabajadores y empresas y con un extenso cometido, igualmente, en materia de formación.

Son funciones del INEM: organizar los Servicios Públicos de Empleo en orden a procurar, pública y gratuitamente, el mejor desarrollo y utilización de los recursos; ayudar a los trabajadores a encontrar empleo y a las empresas a conseguir los trabajadores apropiados a sus necesidades; fomentar la formación del trabajador en estrecha vinculación con la política de empleo, a través de las oportunas acciones de actualización y perfeccionamiento y gestionar y controlar las prestaciones al empleo, (subvenciones y ayudas para el fomento y protección del empleo) y la protección por desempleo.
Todas estas funciones en una primera etapa fueron ejercidas con carácter de monopolio. Con el desarrollo del artículo 137 de la Constitución Española, sobre la articulación territorial del Estado, la gestión de la formación profesional de los desempleados ha pasado durante los años 1991, 1992 y 1993 a cinco Comunidades Autónomas y las organizaciones empresariales y sindicales gestionan, desde 1993, la formación de los ocupados. Por otra parte, algunos programas de empleo han ido pasando, durante estos años, a ser gestionados por las Comunidades Autónomas. Tras la ruptura, en 1994, del monopolio del INEM sobre la intermediación y complementando el desarrollo y la articulación territorial del Estado, alguna Comunidad Autónoma gestionará la intermediación y los programas de empleo acercando la gestión a las fuentes de empleo.

\section{Razones que justifican la implantación de la política de formación}

El artículo 127 del Tratado de la Comunidad Europea, modificado por el Tratado de la Unión aprobado en Maastricht, plantea que la Comunidad ha de desarrollar una política de formación profesional que apoye y complemente las acciones de los Estados para contribuir a desarrollar la cobesión económica y social. En el Memorándum de la Comisión -sobre la formación profesional en la Comunidad Europea para los años 
90 - se indica que el desarrollo de los recursos humanos constituirá un campo prioritario de actuación, para el desarrollo de una politica activa de empleo, para asegurar la preparación de los jóvenes a su vida laboral y para promover la igualdad de oportunidades de los trabajadores para acceder al mercado de trabajo o mantener su ocupación.

Por otra parte, deben considerarse los cambios sustanciales que la economía espanola ha experimentado, tanto cambios en sus estructuras productivas, como organizacionales y ocupacionales. Sin duda, deben destacarse los tres cambios más sobresa- lientes, que se han producido en nuestras estructuras productivas: 1.9$)$ El gran aumento de los servicios y su fuerte dinamismo; 2.9) Los grandes procesos de reestructuración y las amplias inversiones en bienes de capital registrados en todos los sectores, fundamentalmente el sector industrial, que han cambiado la composición productiva de nuestra economía; $\left.3 .^{\circ}\right)$ La creciente importancia de la construcción y las obras públicas que acercan la economía española a la estructura que presentan las economías más avanzadas. Todo ello ha contribuido a modificar la tipología de los métodos de trabajo y a alterar significativamente el contenido de las tareas y hasta la propia organización.

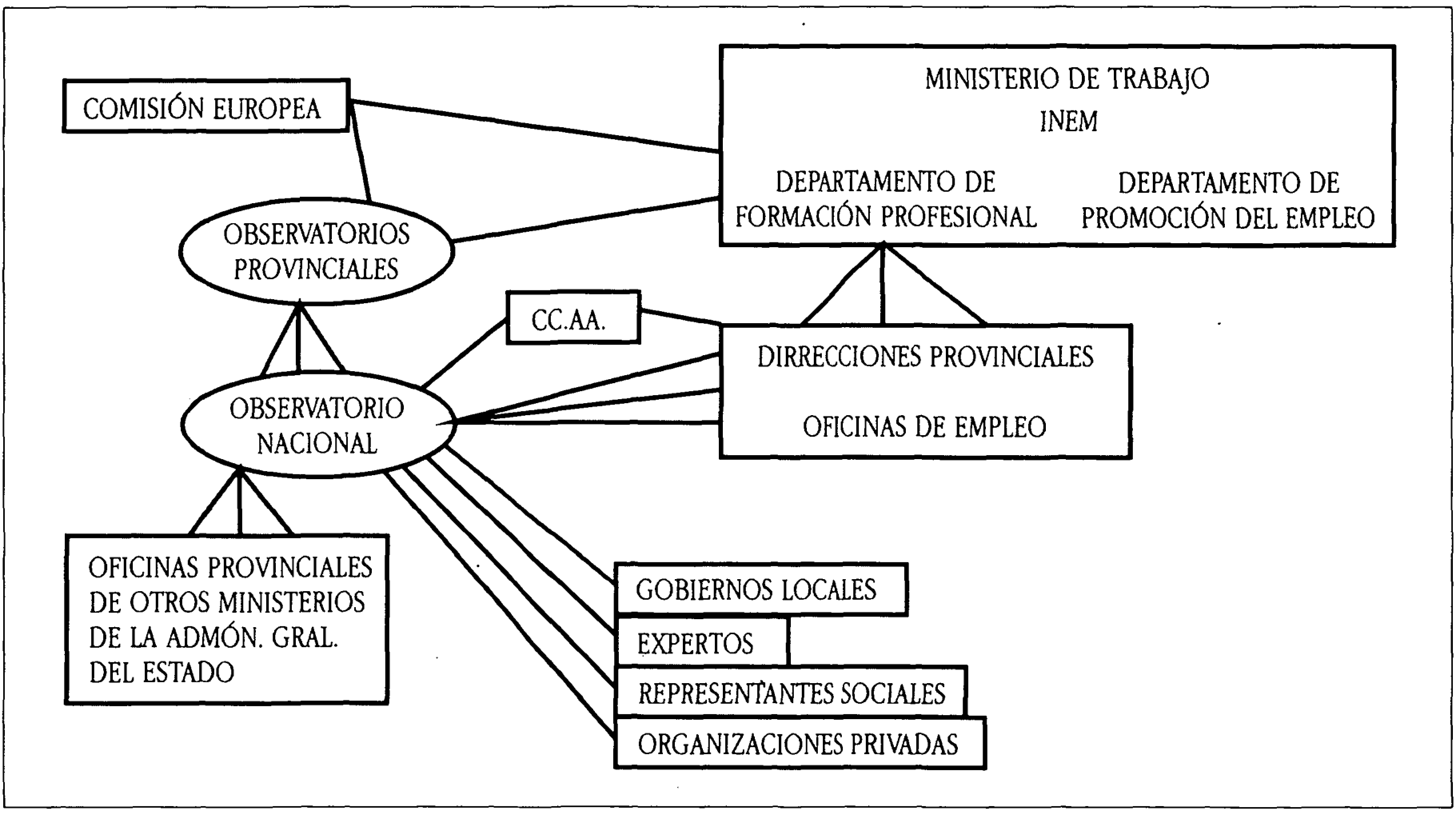

Así pues, entender, por una parte, el origen de los cambios $y$, por tanto, las necesidades derivadas de los mismos constituye la base para garantizar una formación adecuada, dado que la mayor o menor calidad de los recursos humanos disponibles condicionan el desarrollo de cualquier proyecto y la consecución de los objetivos de producción y competitividad en el ámbito productivo; $y$, por otra, que el marco que rodea las organizaciones es dinámico y está ligado fundamentalmente a la competencia y exigencias de la demanda, podemos deducir que dos son los factores que provocan las necesidades de formación: la interacción del ente productor de bienes y/o servicios en un entorno cada vez más universalizado, competitivo y dinámico y la acomodación a las innovaciones del sistema productivo.
Un análisis breve de estas variables nos permitirá ver por donde van las necesidades de la formación. La competencia obliga a una adaptación y exigencias formativas constantes en el terreno de la promoción (con idiomas, conocimiento de nuevas culturas, etc.). La aparición de nuevos productos obliga también a la asimilación de nuevas tecnologías, técnicas gerenciales y comerciales. La aparición en el mercado nacional de productos más competitivos lleva a la creciente necesidad de la productividad y a la introducción de la tercialización. de tareas de los mercados productivos. Las variaciones de la demanda conforman otro elemento dinámico del entomo con la aparición de la existencia de servicios y/o productos que exjgen respuestas formativas para las nuevas actividades y ocupaciones. Y con las innovaciones tecnológicas, surge la necesidad de 
readaptación a los nuevos sistemas productivos y la asimilación de las nuevas tecnologías; además, las modalidades tecnológicas repercuten en el proceso productivo con necesidades de especialización en determinados segmentos.

En conclusión, la formación no sólo obedece a estímulos externos sino también a la propia lógica operativa de la empresa, pues en cualquier momento puede aparecer la necesidad de flexibilizar y readaptar la organización a la misma cadena productiva; por tanto, es necesario el desarrollo permanente de los recursos humanos disponibles en un Estado para conseguir una polivalencia y asi hacer frente a un doble reto: conseguir una respuesta eficaz a los cambios, provenientes del entorno y a los requerimientos del sistema técnico o de gestión y permitir la fácil intercambiabilidad en los distintos puestos de trabajo. Todo ello exige una respuesta activa entendida como respuesta de anticipación a las necesidades, que es lo que se pretende ver con el ejemplo práctico que analizaremos.

\section{Planificación y dirección estratégica}

A pesar de que el entorno de actuación de la Administración Pública es mucho más complicado y ambiguo que el de la empresa privada, ya que en la empresa pública sus objetivos están asociados a las políticas, a los vaivenes coyunturales de la economía o a la utilización anticíclica en años de crisis, no hay que renunciar ni a la planificación y control, ni a los planteamientos estratégicos.

Por una parte, la intensa permeabilidad y complementariedad que progresivamente va adquiriendo la relación de lo público y privado, cada día más aliados y partícipes de un proyecto nacional común en la prestación de servicios, superando la fragmentación y subsidiariedad, exige avanzar necesariamente en la adecuación de las estructuras, instrumentos y procedimientos a los fines y tareas de cada organización y todo ello, en el caso de la Administración, bajo los principios de eficacia, neutralidad, imparcialidad, publicidad y transparencia que rige toda la gestión de los servicios públicos en beneficio de la colectividad, garantizando la máxima eficacia y eficiencia. Hay que situar, pues, los servicios públicos en condiciones de responder adecuadamente a las nuevas y complejas demandas $y$, en este caso, las necesidades del mercado de trabajo, y las demandas de los ciudadanos para coadyuvar con dinamismo a la modernización general del país actualizando los instrumentos de gestión y dirección.
Por otra parte, nuestros servicios, además de continuar con sus funciones garantistas, deben poner cada día más énfasis en la consideración de los ciudadanos como clientes receptores de servicios, superando el antiguo concepto de ciudadano-contribuyente, para darles unos servicios y prestaciones públicas equivalentes en calidad y cantidad a su aportación, poniendo la Administración al servicio de los ciudadanos y de toda la sociedad, sin olvidar la nueva distribución territorial del poder resultante del Estado de las Autonomías, y haciendo de la cooperación con los agentes sociales y las distintas Administraciones Públicas un principio natural y consustancial.

Todo ello tiene una serie de consecuencias que resumimos a continuación:

1.²) La consideración de la organización del trabajo, el papel del directivo como gestor que toma decisiones y es responsable de alcanzar los objetivos, el gestor con el enriquecimiento de las tareas y corresponsable también en la consecución de objetivos.

2.. ) La consideración de la Administración en un Estado descentralizado como el nuestro, como un elemento más que, con otros instrumentos públicos o privados, también defienden el bien común.

3..) El concepto de cliente que adquiere una enorme importancia, porque es el usuario, unas veces como impartidor y otras como receptor de los servicios, el que valora la cantidad y calidad de los mismos.

4. U) Una mayor preocupación por la consecución de los objetivos estratégicos definidos políticamente, el análisis de las programaciones de las acciones y su ejecución y el control de la eficacia y eficiencia de dichas acciones.

\section{Un ejemplo práctico: el uso de la planificación y gestión estratégica en el programa de formación e inserción profesional del INEM}

Dentro del ejemplo, que vamos a presentar, no debemos olvidar que el empleo para todos, que ocupa la atención central de todas las políticas y por tanto la lucha contra el desempleo y la igualdad de oportunidades, supone poder localizar los puntos que presentan deficiencias y sobre los que la actuación es 
prioritaria, así como el tipo de acciones idóneas para lograr la adecuación entre la oferta y la demanda del mercado de trabajo. En España el Observatorio Ocupacional es el que, entre otras funciones, tiene la de analizar las necesidades puntuales de formación a las que hemos hecho referencia.

En el ejemplo siguiente no vamos a explicitar cómo se determina el contenido de los cursos ni cómo se detecta la evolución y tendencia de los distintos sectores productivos o las necesidades de formación, sino cómo se realiza el proceso de la programación y su seguimiento.

Quisiéramos que quedara claro hasta que nivel llega la participación tanto de las Administraciones Públicas como de los interlocutores sociales, empresas, fundaciones, expertos y los mismos receptores de las acciones como una pieza clave del proceso. Asimismo, quisiéramos dejar patente cómo, aunque la programación nace del análisis de las necesidades, éstas se incardinan también en los objetivos estratégicos que priman tanto en la selección de los receptores de la formación, como en la selección de aquellos centros que colaboran para la consecución de dichos objetivos por la adecuación de la formación que imparten y por la calidad de la misma.

Veremos también cómo se realiza un seguimiento de la eficacia y eficiencia del impartidor y receptor de los servicios, bien sean éstos impartidos con medios propios o a través de Centros Colaboradores, valorando también cada una de las acciones realizadas, cada curso y cómo se actúa tras dicha valoración.

Finalmente, a lo largo del ejemplo se verá el papel que juegan tanto los directivos como los responsables de la ejecución del programa de formación.

\section{Proceso}

El Programa de Formación e Inserción Profesional del INEM tiene tres etapas bien diferenciadas:

a) La detección de necesidades.

b) La ejecución de acciones.

c) La evaluación del proceso.

Cada una de estas etapas posee su metodología específica según se detalla a continuación:

\section{Detección de necesidades}

Esta etapa tiene como objetivos específicos: ver la situación y evolución de las actividades económicas y las ocupaciones a nivel provincial y local, detectar los desajustes entre las demandas del mercado y la mano de obra, priorizar la realización de acciones como consecuencia de lo anterior y facilitar parámetros para la posterior evaluación del proceso global.

El desarrollo se lleva a efecto a nivel provincial, desde el mes de enero a finales de octubre de cada año, y participan en ella tanto los técnicos del Observatorio Ocupacional y los responsables de Oficinas de Empleo, por parte del INEM, como expertos e informantes clave de instituciones, organismos y empresas de las actividades económicas más representativas del ámbito territorial como asimismo los agentes sociales.

El proceso metodológico comprende cinco fases:

\section{1) Fase Documental}

La recogida de información afecta tanto a datos internos de la Institución (demandantes de empleo, contrataciones, alumnos, ofertas de trabajo, perfiles ocupacionales de requerimientos del mercado, estudios de sector o monográficos sobre ocupaciones y colectivos, ...) como a otros de carácter externo con incidencia en el mundo laboral (de tipo económico empresarial, poblacional, formativo, ...).

Del análisis de la información obtenida se preseleccionan las ocupaciones sobre las que se va a centrar el resto del proceso, en función de su peso específico en el mercado de trabajo o bien teniendo en cuenta su situación de emergencia o las posibles necesidades de reconversión.

\section{2) Fase de prospección}

El objetivo específico de esta fase es obtener información sobre la previsible evolución de las actividades económicas y ocupaciones, así como de las necesidades de formación, en número y contenidos.

Las técnicas utilizadas son: la consulta a expertos; el sondeo mediante encuesta a los responsables de Oficinas de Empleo y a las Unidades de Promoción y Desarrollo; la entrevista en profundidad a empresas; las reuniones de grupo por actividad económica con informantes clave en el entorno provincial.

\section{3) Fase de análisis}

Tanto los datos cuantitativos como cualitativos obtenidos en las fases anteriores son analizados por el equipo provincial del Observatorio, elaborándose un documento síntesis de Tendencias del Mercado de Trabajo en la provincia y estableciendo las prioridades de formación que las mismas demandan, cuando se trata de provincias cuya gestión de formación es competencia del Estado. 


\section{4) Fase de consenso}

Cuando el INEM gestiona la formación, se celebran en este momento del proceso unas jomadas de trabajo con expertos procedentes de Instituciones, Asociaciones empresariales, organizaciones sindicales, representantes de las CC.AA., etc., en función de las actividades económicas estudiadas, con el fin de dar a conocer los resultados obtenidos y asignar definitivamente las prioridades de formación, de tal manera que los posibles cursos a impartir quedan catalogados con una numeración del 1 al 5 , lo que supone:

1. Prioridad máxima.

2. Potenciar.

3. Mantener.

4. Reducir.

5. Eliminar.

Finaliza esta fase con la emisión de un Informe de Prioridades de Formación Ocupacional de la provincia.

\section{5) Fase de difusión}

El informe de Tendencias del Mercado de Trabajo en la provincia se pone a disposición no sólo de los agentes que han participado en el proceso en sus diferentes niveles sino también a la de las Instituciones, Universidades y empresas de la provincia, así como a la de los responsables del empleo y la formación en la Comunidad Autónoma respectiva.

El informe de las Prioridades de Formación Ocupacional de las provincias se facilita a los Centros e Instituciones que colaboran con el INEM y se realiza de todos ellos un resumen de carácter nacional que recopila lo correspondiente a las provincias en que la gestión de la formación ocupacional es competencia del INEM.

\section{Ejecución de acciones}

Esta actividad tiene dos aspectos que se complementan.

a) La programación.

b) El seguimiento y control.

\section{Programación}

La ejecución de acciones de formación ocupacional está condicionada a los Presupuestos anuales aprobados para tal fin, si bien es cierto que la propuesta de la cuantía presupuestaria está formulada en base a distintos factores entre los que cuentan los programas operativos cofinanciados por el Fondo Social
Europeo que han sido aprobados por Decisión de la Comisión Europea para España, aspectos del análisis de la evolución de las ocupaciones, evolución del empleo, etc.

Con base en los Presupuestos aprobados, se establecen los objetivos de formación ocupacional en número de alumnos y mediante el indicador adistribuidor único (fómula en la que intervienen factores del empleo) se realiza la distribución de los objetivos y el correspondiente presupuesto a las distintas unidades de gestión, direcciones provinciales del INEM y Comunidades Autónomas a las que se ha transferido la gestión de la formación ocupacional del Plan Nacional de Formación e Inserción Profesional.

Establecidas estas premisas, los pasos para cumplir los objetivos estratégicos de formación se concretan:

- A las Comunidades Autónomas a las que se les ha transferido la Gestión de la Formación Ocupacional, se les remiten los créditos presupuestarios que les corresponda, con el fin de que, con base en las competencias asumidas, ejecuten los objetivos del PLAN FIP que les han sido asignados.

Como responsables directos, estas Comunidades Autónomas instrumentan los procedimientos de programación y seguimiento y control de la Formación Ocupacional regulada por el Real Decreto 631/1993, de 3 de mayo, y Órdenes Ministeriales que lo desarrollan y les afecta.

* A las Direcciones Provinciales del INEM en las provincias de Comunidades Autónomas a las que no ha sido transferida la Gestión de la Formación Ocupacional, se les comunican los "objetivos" que les corresponden, se les dan instrucciones de Programación de cursos de Formación Ocupacional y se les remiten los créditos presupuestarios correspondientes para la ejecución de las acciones formativas.

Estas Direcciones Provinciales del INEM, como ejecutoras del PLAN FIP, realizan una propuesta de programación de cursos de Formación Ocupacional basándose en los análisis de la fase anterior y la remiten informada por la correspondiente "Comisión Ejecutiva Provincial", en la que están representadas las Fuerzas Sociales (patronal y sindicatos) y la Administración.

Esta propuesta de programación se elabora considerando fundamentalmente los aspectos siguientes:

a) Prioridades provinciales, establecidas en el análisis de la evolución del mercado de trabajo.

b) Objetivos estratégicos de Formación Ocupacional y Programas Operativos del Fondo Social Europeo.

c) Evaluación de Cursos y Centros en la ejecución de programaciones de años anteriores.

d) Informes de control de seguimiento de programaciones de años anteriores. 
e) La oferta formativa de los Centros Colaboradores del INEM, materializada en las solicitudes de programación.

f) La oferta formativa de los Medios Propios del INEM. Capacidad y especialidades de sus propios Centros de Formación Ocupacional.

Una vez estudiadas las propuestas de programación de las distintas Direcciones Provinciales, la Subdirección General de Gestión de Formación Ocupaciona elabora y aprueba la Programación Nacional de Cursos del PLAN FIP, previo informe de la Comisión Ejecutiva Nacional, en la que están representados la patronal, los sindicatos y la Administración, y que oportunamente comunica a cada Dirección Provincial para su puesta en marcha.

\section{Seguimiento y control}

Además del seguimiento sistemático que cada Dirección Provincial realiza de las acciones de formación que va desarrollando, y que le permite ir corrigiendo defectos y errores que puedan surgir en la ejecución de la programación, la Subdirección General de Gestión de Formación Ocupacional establece un Plan anual de control de Subvenciones que permite detectar anomalías en la mencionada ejecución de Programación con el fin de, por una parte, tomar las medidas administrativas que corresponda y, por otra, que sirva de información para evitar que ocurra de nuevo en sucesivas ediciones.

Este Plan de control consiste, en términos generales, en fijar a cada Dirección Provincial, a tenor de su volumen de programación, un número de cursos a nauditar. con el fin de realizar un análisis del cumplimiento de los requisitos de carácter formativo de desarrollo de los cursos y otro de los costes de la actividad desarrollada y verificar que la subvención abonada al Centro está debidamente justificada.

Por otra parte, la Intervención General de la Administración del Estado anualmente solicita al INEM la Programación realizada y por muestreo establece los cursos y Centros a los que realizará una auditoría. Una vez realizadas estas auditorías remiten al INEM las correspondientes actas en las que se recoge lo detectado por la IGAE, con base en las cuales se adoptan las medidas administrativas necesarias.

\section{Evaluación del proceso}

La evaluación de la formación tiene como objetivo obtener una valoración global de cada una de las especialidades formativas, así como, analizando sucesivas programaciones, ver la adecuación de los Centros por especialidades y por factores dentro de las mismas y se efectúa en cuatro momentos diferentes:
1) Durante el curso

El centro impartidor es visitado por técnicos del INEM los cuales cumplimentan un impreso en el que se evalúan los programas de conocimientos y prácticas, las instalaciones, los equipos, el material de consumo, los medios didácticos y el profesorado, con el objetivo de conseguir un conocimiento continuo del desarrollo de los cursos.

2) Al finalizar el curso

En este caso se realiza con el fin de conocer tanto aspectos globales de la acción formativa no relacionados de un modo directo con el proceso de formación (las expectativas de empleo o las relaciones del Centro con su entorno) como aspectos concretos del proceso en su momento final, es decir: las pruebas de evaluación de los alumnos o la adecuación de los contenidos a los objetivos formativos propuestos.

\section{3) Transcurrido un año desde la finalización del curso}

El objetivo de esta acción es conocer la inserción profesional del alumno.

Las técnicas utilizadas para este seguimiento de la inserción son tres:

- Dado que el INEM posee un registro de más del $90 \%$ de la contratación que se efectúa en el país, cada número identificador del alumno se rastrea en dicha base de contratos durante el año siguiente a la finalización del curso, con objeto de conocer si hubo contratación, cuánto tiempo transcurrió desde la finalización del curso hasta la misma, qué tipo de contrato/s ha recibido y la duración del/los mismos.

- Para la obtención de otros datos cualitativos tales como motivación para realizar el curso, expectativas, sistemas de búsqueda de empleo, si el trabajo conseguido está relacionado con el curso o no, si ha continuado su formación, etc., se envía anualmente una encuesta a una muestra de antiguos alumnos con un nivel de confianza del 95\% en cada provincia.

- Finalmente, se establecen dos grupos de control: uno de parados que recibieron formación y otro, idéntico en fecha, edad, sexo y nivel académico, de demandantes de empleo que no asistieron a cursos, con el fin de poder comprobar la incidencia de la formación sobre el empleo.

4) Anualmente, desde los Servicios Centrales del INEM y teniendo en cuenta el seguimiento y control de la ejecución y los resultados de evaluación de los tres momentos anteriores, se realiza una evaluación final de cumplimiento de los objetivos estratégicos y se elabora la justifica- 
ción de los Programas Operativos cofinanciados por el Fondo Social Europeo.

\section{Efectos del proceso de planificación y gestión estratégica}

La implantación de la planificación y gestión estratégica en el Programa de Formación e Inserción Profesional ha supuesto la resolución de problemas de gestión, de equidad territorial, de eficacia de las acciones, ..., si bien es susceptible de mejora para conseguir día a día resultados más en consonancia con los objetivos de un servicio público de empleo, de suministrar a los usuarios del mismo una mayor y más especializada atención.

Las peculiaridades de organización del Estado español exigen un esfuerzo especial de coordinación entre todas las actuaciones autonómicas, sobre todo teniendo en cuenta que, en cuestiones de formación ocupacional, son cinco las Comunidades Autónomas con competencias transferidas de gestión de la misma. Por ello, la homogeneización del proceso de detección de necesidades de formación y las actuaciones coordinadas son esenciales para garantizar una atención equitativa a los usuarios.

El proceso de planificación y gestión estratégica ha tenido, además, la virtualidad de acercar la Administración del Estado a las necesidades concretas de los ámbitos locales, ya que el realizar la detección desde las provincias y participar en ella instituciones, empresas, oficinas de empleo, expertos, etc., con representatividad no sólo profesional sino también geográfica, permite conseguirlo.

En cuanto a los Centros que colaboran con el INEM en la impartición de acciones, el hecho de establecer un sistema de selección y seguimiento de los mismos ha permitido superar los problemas iniciales de adecuación de medios materiales a los contenidos formativos, teniendo en. cuenta la importancia de esto ya que la formación ocupacional es, por su propia concepción, eminentemente práctica aunque con la teoría necesaria para la comprensión del trabajo concreto que se realiza. Por su parte, además, los Centros Colaboradores han empezado a entrar en la dinámica de formación ocupacional para el empleo y cada vez son más los que se preocupan y gestionan la salida laboral de sus ex alumnos.

No debe olvidarse que la dinamización del proceso y la participación en él de todos los afectados por el mismo, ha supuesto la concienciación del papel de la formación, no sólo en cuanto potenciadora de las posibilidades de inserción laboral o de mantenimiento del puesto de trabajo, sino también como portadora de valores añadidos para los usuarios tales como satisfacción personal, autoconfianza, mayor participación en la búsqueda activa de empleo, ...

De otro lado, a través de la mayor participación y compromiso de todos los agentes intervinientes y del acercamiento al ámbito local, se ha obtenido como resultado una mejor selección del personal, de los Centros y de los colectivos destinatarios y todo ello sin aumentar en los últimos años el presupuesto destinado a formación ocupacional de personas en desempleo.

Si se mide, además, la eficacia del proceso en términos de inserción profesional de los usuarios, los resultados son positivos ya que en los últimos seis años se ha incrementado paulatinamente la colocación de alumnos en 20 puntos porcentuales, aunque con un ligero declive en los años 1992 y 1993 debido a las dificultades coyunturales por las que atravesó el mercado de trabajo. Por otra parte, las posibilidades de acceder a un puesto de trabajo son superiores en un $18 \%$ para los que han recibido formación ocupacional que para los que carecen de ella y con mayor incidencia (1,5\%) en las zonas más deprimidas que en las regiones con más elevado desarrollo económico.

No obstante lo anterior, el sistema tiene cuestiones pendientes de resolución satisfactoria. Es necesario conseguir una mayor incardinación de las Comunidades Autónomas con transferencia de la gestión de la formación en el proceso, de tal modo que el mismo adquiera consistencia en todo el territorio nacional, con el fin de evitar discriminaciones entre los clientes según el ámbito geográfico.

Es necesario que la filosofía subyacente al proceso vaya calando cada vez más en los Centros Colaboradores para que su participación sea plena y acorde en todas las fases del mismo, siendo necesario tambien ampliar la red de impartidores buscando la colaboración de entidades muy especializadas para cursos específicos.

Asimismo, en ciertos territorios queda aún por resolver satisfactoriamente la carencia de entidades de formación que cubran las necesidades específicas de la zona.

En conclusión, el sistema de planificación y gestión estratégica ha permitido resolver muchos de los problemas planteados para conseguir que la formación ocupacional sea una de las medidas de ajuste del desequilibrio entre la oferta y la demanda de mano de obra en el mercado de trabajo si bien, como todo proceso, ha de irse perfeccionando con la experiencia y adaptándose a los requerimientos dinámicos de la inserción laboral. 
Novembro/Dezembro 1997

Vol. 31 n. 96 ISSN $0034-7612$

Periocidad bimestral

Revista da Escola Brasileira de Administração

Pública da Fundaçāo Getulio Vargas

\section{EXPEDIENTE}

Director: Bianor Scelza Cavalcanti

Conselho Editorial: Anna Maria de Souza Monteiro Campos, Aspásia Camargo, Bernardo Kilksberg. Heitor Chagas de Oliveira, J. J. R. Fraústo da Silva, Marcos Cintra Cavalcanti de Albuquerque, Paulo Roberto Motta, Sonia Maria Fleury Teixeira, Tânia Fischer

Redatora: Deborah Moraes Zouain

\section{EDITORA FUNDAÇĀO GETULIO VARGAS}

Editora executiva: Alzira Alves de Abreu

Coordenação editorial: Cristina Mary Paes de Cunha

Copidesque: Maria Izabel Penna Buarque de Almeida

Editoração electrônica: Jayr Ferreira Vaz e Simone Ranna Revisäo: Aleidis de Beltran, Fatima Caroni e Mauro Pinto de Faria Produção gráfica: Helio Lourenço Netto

Gerente comercial: Carlos Hamilton Rocha

Supervisora de marketing direto: Maria Cristina Carrez

Capa: Tira linhas studio

Índices nos quais a Revista de Administração Pública é citada: Bibliographie Latinoaméricaine d'Articles, n. ${ }^{9} 22,1987$;

Handbook of Latin American Studies: Social Sciences, v. 41, 1979; Indices de Ciências Sociais/luperi, v. 9, 1989; International Bibliography of the Socials Sciences Economics; Sumários Correntes Brasileiros: Ciências Sociais e Humanas

CORRESPONDÊNCIA REDATORIAL

Inclusive originais de artigos (três vias):

REVISTA DE ADMINISTRAÇĀO PÚBLICA

Praia de Botafogo, 190 - sala 426-

CEP 22253-900

CORRESPONDÊNCIA COMERCIAL

FUNDAÇĀO GETULIO VARGAS/EDITORA

Caixa Postal 62.591 - CEP 22252-970

Rio de Janeiro, RJ, DDG: (0800) 21-7777

e-mail: assine @ fgv.br

FUNDAÇÃO GETULIO VARGAS

LIVRARIA J. CARNEIRO FEUPE

Praia de Botafogo, 188

Rio de Janeiro, RJ - CEP 22253-900

Tel.: (021) 536-9105

LIVARARIA FARIA LIMA

Av. Nove de Julho, 2.029, Sao Paulo, SP

Tel.: (011) 281-7875

Palavras do director

ARTIGOS

Um novo paradigma para as instituições públlcas de pesquisa

Anne-Marie Maculan e Deborah Moraes Zouain

O empresarialismo dos governos locais na gestão urbana:

breves reflexōes sobre a origem, a evolução e o contexto

deste concelto no Brasil

Antônio Sérgio Araújo Fernandes

O Estado e as ONGs: uma parceria complexa

Maurício Serva
Associativismo como instrumento de desenvolvimento dos governos locais: a experiêncla brasileira e de outros países

Diogo Lordello de Mello

O transporte urbano como política sócio-econömica: uma perspectiva latino-americana

Enrique Saravia

À busca de um novo modelo de segurança para o hemisfério americano

Artur Luiz Santana Moreira

ESPAÇO INTERNACIONAL

Elementos condicionantes para el éxito de los procesos de regionalización

Alejandro Santibañez Handschuh

DEPOIMENTOS

Capacitaçāo de administradores para as organizaçōes sem fins lucrativos

Fátima Bayma

Liderança para o amanhã: as questōes da educação internacional

Dorothy S. Moore.

Tradução: Valderez F. Fraga

Curso de Pós-Graduação em Administração Esportiva (CPAES)

Equipe de coordenação: Deborah Moraes Zouain e Luiz Alberto Batista

Apóio técnico-acadêmico: José Antonio Barros Alves

Curso de Pós-Graduação em Gestāo de Recursos Huma-

nos (CEGERH)

Curso de Pós-Graduação em Logística Empresarial

Curso de Pós-Graduaçāo em Gerência Avançada de Negócios

RESENHA BIBLIOGRÁFICA

A última palavra em poder - reinvenção executiva para

lideres que precisam fazer o impossivel acontecer.

Tracy Goss. Rio de Janeiro, Rocco, 1997. 311 p.

Sylvia Constant Vergara

SEÇŌES ESPECIAIS

Programa de Estudos em Gestão Social

Escritório-modelo de elaboração de projetos comunitários

Fernando G. Tenório (coord.), Ângela M. M. Sampaio, Augusto P. G. Cunha, Elvira C. F. Ventura, Gylcilene R. Storino, José A B. Alves, Luiz F. F. Ferreira, Martha M. F. da Costa, Patricia N. da Cruz e Zugleima del C. Hidalgo

Programa de Estudios en Gestión Social: Proyecto Ciudadanía y Municipalidad

Zugleimia del Carmem Hidalgo

Programa de Estudos e Pesquisas em Reforma do Estado e Governança

O balanço das reformas na América Latina

5 Sonia Fleury

A conjuntura das escolhas públicas

O bureaucratic drift: novos fatos sobre a economia das medidas provisorias

7 Jorge Vianna Monteiro

Producão e desenvolvimento nos estados e municíplos Indice das fontes

Istvan Karoly Kasznar
41 